\title{
Experience Related Impacts on Jump Performance of Elite and Collegiate Basketball Players; Investigation on Force-Time Curvature Variables
}

\author{
Javad Sarvestan ${ }^{1}$, Mahdi Cheraghi ${ }^{2}$, Elham Shirzad ${ }^{3}$ and Zdenek Svoboda ${ }^{1}$ \\ ${ }^{1}$ Palacky University Olomouc, Faculty of Physical Culture, Department of Natural Sciences in Kinanthropology, Olomouc, Czech Republic, ${ }^{2}$ National \\ Olympic and Paralympic Committee of Iran, Tehran, Iran, ${ }^{3}$ University of Tehran, Faculty of Physical Education and Sport Sciences, Department of \\ Health and Sports Medicine, Tehran, Iran
}

\begin{abstract}
Force-Time (F-T) curve variables of the vertical jump are known as contributing factors in jumping height. Experience-related differences might also have impacts on kinetic and kinematic outputs of athletes'jump. The aim of this study was to investigate the correlation between F-T curve variables with jump height $(\mathrm{JH})$ and observe the differences between elite and collegiate basketball players. With institutional ethics approval, 12 elites (24.3 \pm 5.9 years, $195.4 \pm 23.1 \mathrm{~cm}, 89.1 \pm 15.2 \mathrm{~kg}, 13.6 \pm 2.3$ years' experience) and 12 collegiate $(21.6 \pm 2.5$ years, $183.2 \pm 6.1 \mathrm{~cm}$, $75.3 \pm 9.5 \mathrm{~kg}, 9.1 \pm 1.8$ years' experience) male basketball players participated in this study. Correlation between F-T variables -included time, force, velocity, power and modified reactive strength (MRSI) - in the eccentric and concentric phases and JH was studied. Outcomes portrayed that concentric Relative Peak Force $(r=0.71)$, Relative Peak Power ( $r=0.83)$, Peak Velocity $(r=0.99)$ and MRSI $(r=0.71)$ in elite players, and concentric Relative Peak Force $(r=0.79)$, Average Power ( $r=65)$, Relative Peak Power $(r=0.81)$, Peak Velocity $(r=0.98)$ and MRSI $(r=0.83)$ in collegiate players were significantly correlated with $\mathrm{JH}$. Absolute and relative measures of power and force are shown to have a vital role in jump performance of basketball players. MRSI, which is defined as an explosiveness measurement in athletes, could be accounted as of jump performance criteria. Focusing on selected factors described above in training programs could enhance athlete jump performance, particularly in basketball society.
\end{abstract}

Key words: countermovement jump, F-T curve, power, modified reactive strength index

\section{Introduction}

Lower limb muscle strength and power are of crucial criterion for achieving a prosperous performance among athletes (Canavan \& Vescovi, 2004; Markovic, 2007). Vertical jumps are the most commonly used tests for examination of lower limbs' strength and power (Jidovtseff, Quievre, Nigel, \& Cronin, 2014; Laffaye, Wagner, \& Tombleson, 2014). Previous studies focused on athletes' vertical jump performance, particularly obtained jump height (JH) (Jidovtseff et al., 2014; Laffaye et al., 2014; Sarvestan, Cheraghi, Sebyani, Shirzad, \& Svoboda, 2018; Smith, Roberts, \& Watson, 1992). Furthermore, vertical jumps are also employed to assess and evaluate the explosive strength and power of the lower extremity in order to forecast and monitor the motor performance of athletes in several sports such as soccer, handball, volleyball, basketball, and sprinting (Carlock et al., 2004; Jiménez-Reyes \& González-Badillo, 2011). Former studies are mostly assessed lower extremity power by performing a countermovement jump (CMJ) (Kollias, Hatzitaki, Papaiakovou, \& Giatsis, 2001; Laffaye et al., 2014; Sarvestan et al., 2018; Slinde, Suber, Suber, Edwén, \& Svantesson, 2008). The CMJ is a type of vertical jump that is considered as the most useful test to assess neuromuscular coordination of athletes (Claudino et al., 2017) as well as neuromuscular and biomechanical features related to lower

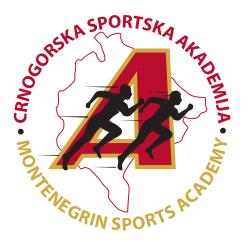

Correspondence:

Palacky University Olomouc, Faculty of Physical Culture, Department of Natural Sciences in Kinanthropology, Olomouc, Czech Republic E-mail: javad.sarvestan01@upol.cz 
extremity dynamics (Claudino et al., 2017; Kollias et al., 2001). Vertical jump tests are generally used for various aims such as assessing the force and power of elite athletes who regularly participate in competitions (Cormie, McGuigan, \& Newton, 2010). $\mathrm{CMJ}$ was also utilized in order to assess the effect of fatigue on performance (McGinnis et al., 2016).

$\mathrm{CMJ}$ includes rapid eccentric, and subsequently, concentric activity of muscle that is termed as stretch-shortening cycle (SSC). The SCC nature is known as rapid concentric contraction after aquick eccentric contraction that increases stored energy and muscle activity (Cormie, McBride, \& McCaulley, 2009; Cormie et al., 2010). Pre-activation, activating muscle structures prior to implementation, rate of change of muscle length compared with rate of change in the tendon, and role of stretching reflexes are the other effective aspects in SSC. lower extremity power during the concentric phase is of factors that positively affect SSC, and in turn increase the jump height by increasing the center of mass vertical velocity. Sufficient time for producing and transferring force to the skeletal system is an extremely effective mechani$\mathrm{sm}$ in SSC. The eccentric phase of SSC provides agonist muscles with sufficient time to generate significant force, and also provides sufficient time to the structures to reach significant stiffness (Jiménez-Reyes \& González-Badillo, 2011). Previous studies indicated that athletes exhibit better performance in CMJ compared with that in squat jump (SJ) (Bobbert, Gerritsen, Litjens, \& Van Soest, 1996; Bosco, Viitasalo, Komi, \& Luhtanen, 1982; Cormie et al., 2010; Laffaye et al., 2014; Sheppard, McGuigan, \& Newton, 2008), which could be due to SSC nature and especially because of the eccentric phase effect that exists in CMJ. Predominantly, studies on CMJ are generally focused on the concentric phase and there is a paucity of studies on the eccentric phase.

It has been pointed out that CMJ Force-Time (F-T) curvature variables, such as time, force and derived components could reveal an in-depth information on athletes' jump performance (Laffaye et al., 2014). Training level and neuromuscular adaptation, additionally, are brought up as of effective parameters on F-T curve shape (Cormie, McBride, \& McCaulley, 2008). Given the nature of SSC, cooperation of elastic contractile elements in particular, it has been speculated that biomechanical variables portray vital information on jump performance. Power and force measures, in addition to velocity, are of mechanical parameters surveyed in former investigations (Cormie et al., 2008; Jidovtseff et al., 2014; Laffaye et al., 2014; Sarvestan et al., 2018).

Scientific literature indicated that achieving relative peak power affects ultimate height during CMJ in both volleyball and basketball (Markovic, 2007; Riggs \& Sheppard, 2009). It has, also, mentioned that relative peak power and average power are significantly correlated with ultimate height in CMJ performance (Riggs \& Sheppard, 2009). Relative power measure, which is normalized with body weight, is suggested that individual relative power significantly impacts performance in order to reach the ultimate possible jump height. However, other force-time (F-T) curve variables derived from CMJ, such as peak power $(\mathrm{PP})$, peak velocity $(\mathrm{PV})$, relative force $(\mathrm{RF})$, average force $(\mathrm{AF})$, and peak force $(\mathrm{PF})$ were indicated as effective variables in concentric and eccentric phases of CMJ by several studies (Cormie et al., 2009; Cormie et al., 2010; Laffaye et al., 2014; Riggs \& Sheppard, 2009; Sarvestan et al., 2018). However, number of studies that consider the experience-related differences in vertical jump performances among elite and sub-elite players, particularly in basketball players, are scarce. A description of these differences would aid coaches and fitness practitioners in planning optimal exercise program for athletes. To this end, and based on background covered above, the main objective of the present study involves assessing differences in F-T curve variables describing CMJ jump performance between elite and collegiate basketball players and assessing the relationship between F-T curve variables and height of CMJ.

\section{Methods}

Participants

Twenty-four elite $(n=12,24.3 \pm 5.9$ years, $195.4 \pm 23.1 \mathrm{~cm}$, $89.1 \pm 15.2 \mathrm{~kg}, 13.6 \pm 2.3$ years' experience) and collegiate $(\mathrm{n}=12$, $21.6 \pm 2.5$ years, $183.2 \pm 6.1 \mathrm{~cm}, 75.3 \pm 9.5 \mathrm{~kg}, 9.1 \pm 1.8$ years' experience) basketball player have participated in this study. Participants were acquainted with the purpose of the study and methods in details, and also, signed an informed consent form to participate in the study.

\section{Instruments and procedures}

After a 10-minute dynamic warm up, and prior to data collection, manner of performing CMJ was explained by an expert trainer. Afterwards, each participant performed CMJ, interspersed by $1 \mathrm{~min}$ intervals of rest to prevent fatigue, to a self-selected depth on force platform (Kistler, 9290AD, Winterthur, Switzerland), which measured three dimensional components of GRF at a frequency of $1000 \mathrm{~Hz}$ (Sarvestan et al., 2018). The CMJ performance authenticated by trainer and each participant performed three CMJs. The effect of arm swing on jump height is reviewed more than four decades and previous studies indicated that arm swings, during takeoff phase, can increase vertical velocity of center of mass for 10\% (Markovic, 2007). For this reason, to prevent the effect of arm swing and focusing on lower extremity, participants put their hands on their waist (Figure 1).

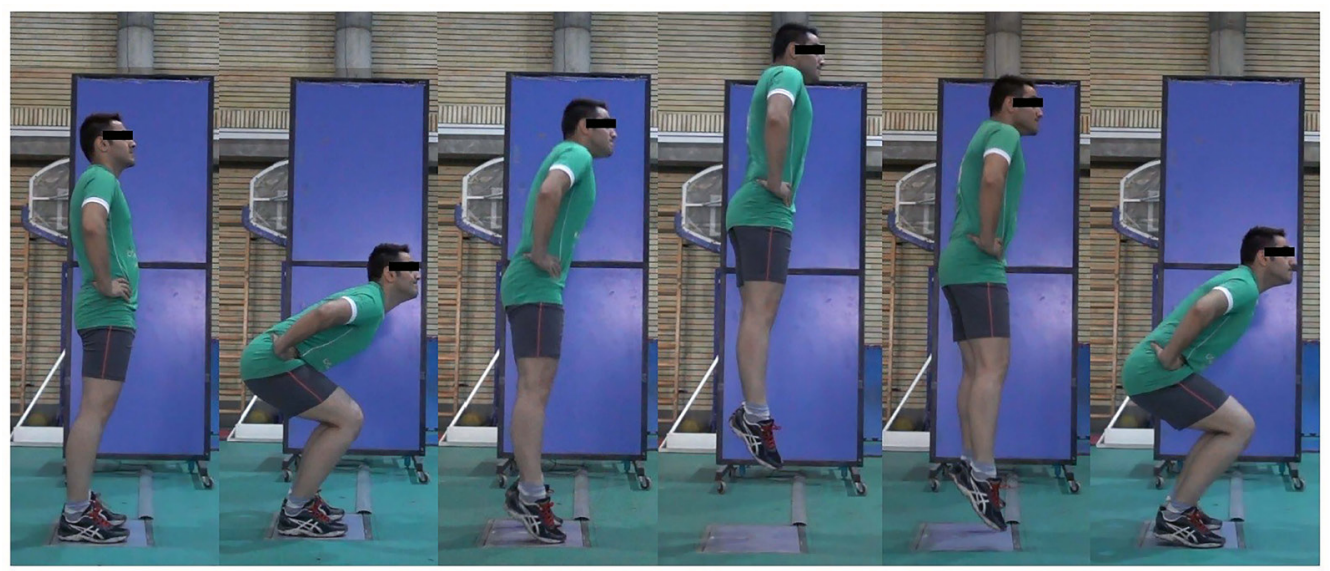

Figure 1. Manner of Performing Countermovement Jump - Akimbo Style 


\section{Data analyses}

Values measured by a force platform was analyzed in Excel 2016 software. After achieving the F-T curve through the force platform, data analysis of F-T curve variables was accomplished in three phases, namely the unweighting phase, eccentric phase, and concentric phase. A few studies divided CMJ into the following three phases: unweighting, eccentric, and concentric phases (Laffaye et al., 2014; Pupo, Detanico, \& Santos, 2012; Sarvestan et al., 2018); however, in this study, CMJ was divided into eccentric and concentric phases to review the special impact of each F-T curve variables on $\mathrm{JH}$.

Instantaneous COM velocity was calculated by dividing the vertical force (excluding body weight) by the body mass and then integrating the product by using the trapezoid rule (Sarvestan et al., 2018). Instantaneous power was calculated by multiplying vertical force and velocity data at each time point, and COM displacement was determined via double integration of the vertical force data (McMahon, Rej, \& Comfort, 2017; Owen, Watkins, Kilduff, Bevan, \& Bennett, 2014). Eccentric (E) and concentric (C) peak force (PF), peak power (PP), and peak velocity $(\mathrm{PV})$ were defined as the maximum values attained during the eccentric and concentric phases. All kinetic data were divided by the body mass to allow a normalized comparison of these data between athletes. The JH was derived from the vertical velocity at take-off(Moir, 2008).

\section{Statistical analyses}

Kolmogorov-Smirnov test was employed to check normality of data distribution. A paired sample T-Test was also used to determine F-T curve variables' differences among elite and collegiate players. Pearson Product Moment correlations were performed to determine the relationships among eccentric and concentric phase's variables with ultimate jump height. The level of significance was set as $\mathrm{p}<0.05$. This analysis was performed using the SPSS software (version 22.0, IBM Corp., Armonk, NY). In order to increase statistical power of results and prevent the effect of small sample size on the outcomes, all the variables for the entire participants were assessed after the intergroup analysis.

\section{Results}

Descriptive measures of F-T curve variables, MRSI and JH and the differences between elite and collegiate players are illustrated in Table 1.

Table 1. Descriptive measures of all variables in eccentric and concentric phases and differences between elite and collegiate players

\begin{tabular}{|c|c|c|c|c|c|c|}
\hline \multirow{2}{*}{\multicolumn{2}{|c|}{ Variables }} & \multicolumn{2}{|c|}{ Descriptive Measures (Mean \pm SD) } & \multicolumn{3}{|c|}{ Differences } \\
\hline & & Collegiate & Elite & $\mathbf{t}$ & df & Sig. (2tailed) \\
\hline \multirow{6}{*}{ Time (s) } & ETT & $0.203 \pm 0.13$ & $0.184 \pm 0.09$ & 0.92 & 11 & 0.44 \\
\hline & CTT & $0.537 \pm 0.13$ & $0.470 \pm 0.08$ & -3.94 & 11 & $0.02 \dagger$ \\
\hline & $\mathrm{TtE}$ & $0.280 \pm 0.17$ & $0.291 \pm 0.22$ & 0.16 & 11 & 0.82 \\
\hline & $\mathrm{TtC}$ & $0.483 \pm 0.13$ & $0.475 \pm 0.08$ & -0.23 & 11 & 0.74 \\
\hline & TtEPP & $0.482 \pm 0.18$ & $0.475 \pm 0.04$ & -0.22 & 11 & 0.71 \\
\hline & TtCPP & $0.975 \pm 0.11$ & $0.901 \pm 0.09$ & -5.74 & 11 & $0.01 \neq$ \\
\hline \multirow{4}{*}{$\begin{array}{l}\text { Force } \\
(\mathrm{N})\end{array}$} & EPF & $885 \pm 109$ & $903 \pm 386$ & 0.11 & 11 & 0.89 \\
\hline & CPF & $2041 \pm 346$ & $2165 \pm 726$ & 1.59 & 11 & 0.12 \\
\hline & ERPF (N/kg) & $11.76 \pm 0.04$ & $10.54 \pm 0.09$ & -1.73 & 11 & 0.10 \\
\hline & CRPF $(\mathrm{N} / \mathrm{kg})$ & $27.16 \pm 4.35$ & $24.28 \pm 5.64$ & 2.84 & 11 & $0.04 \dagger$ \\
\hline \multirow{3}{*}{$\begin{array}{c}\text { Velocity } \\
(\mathrm{m} / \mathrm{s})\end{array}$} & EPV & $0.58 \pm 0.06$ & $0.65 \pm 0.10$ & 1.02 & 11 & 0.28 \\
\hline & CPV & $1.37 \pm 0.19$ & $1.60 \pm 0.23$ & -6.12 & 11 & $0.01 \neq$ \\
\hline & EPP & $816 \pm 149$ & $849 \pm 201$ & 0.81 & 11 & 0.52 \\
\hline \multirow{7}{*}{$\begin{array}{l}\text { Power } \\
\text { (W) }\end{array}$} & CPP & $4697 \pm 1742$ & $5118 \pm 3047$ & 1.92 & 11 & $0.01 \neq$ \\
\hline & EAP & $461 \pm 79$ & $423 \pm 41$ & -1.03 & 11 & 0.27 \\
\hline & CAP & $1267 \pm 593$ & $1620 \pm 819$ & 4.96 & 11 & $0.01 \neq$ \\
\hline & $\operatorname{ERPP}(\mathrm{W} / \mathrm{kg})$ & $10.88 \pm 1.93$ & $9.54 \pm 1.82$ & -1.81 & 11 & 0.09 \\
\hline & CRPP $(\mathrm{W} / \mathrm{kg})$ & $62.59 \pm 18.74$ & $57.51 \pm 21.38$ & -4.08 & 11 & $0.02+$ \\
\hline & IRSI & $0.361 \pm 0.015$ & $0.419 \pm 0.018$ & 7.16 & 11 & $0.00 \neq$ \\
\hline & $(\mathrm{m})$ & $0.389 \pm 0.052$ & $0.441 \pm 0.037$ & -6.81 & 11 & $0.01 \neq$ \\
\hline
\end{tabular}

Legend: Eccentric Total Time (ETT), Concentric Total Time (CTT), Time to Eccentric (TtE), Time to Concentric (TtC), Time to Eccentric Peak Power (TtEPP), Time to Eccentric Peak Power (TtCPP), Eccentric Peak Force (EPF), Concentric Peak Force (CPF), Eccentric Relative Peak Force (ERPF), Concentric Relative Peak Force (CRPF), Eccentric Peak Velocity (EPV), Concentric Peak Velocity (CPV), Eccentric Peak Power (EPP), Concentric Peak Power (CPP), Eccentric Average Power (EAP), Concentric Average Power (CAP), Eccentric Relative Peak Power (ERPP), Concentric Peak Power (CRPP), Modified Reactive Strength Index (MRSI), Ultimate Jump Height (JH).

† Significantly different $(p \leq 0.05) ; \neq$ Significantly different $(p \leq 0.01)$

The results indicate that $\mathrm{CMJ}$ execution time was significantly higher in collegiate players in comparison with elites, particularly in concentric phase $(0.067 \mathrm{~s})$. Consequently, elites could reach to CPP significantly sooner than collegiate players $(0.074 \mathrm{~s})$ in their jumps. Although elite players produced relatively higher force measures, the amounts of CRPF were significantly higher in collegiate players $(p=0.04)$. Similarly, albeit CPP $(p=0.01)$ and CAP $(\mathrm{p}=0.01)$ measures were significantly higher among elite players, collegiate players produced significantly more CRPP values $(\mathrm{p}=0.02)$. Recorded MRSI measures were meaningfully more in elites in comparison with collegiate players (14\%), and elites jumped significantly higher compared with collegiate players $(\approx 5 \mathrm{~cm})$. Furthermore, as could be seen in Figure 2, elites bent approximately 8 centimeters more than collegiate players $(\mathrm{p}=0.00)$. 


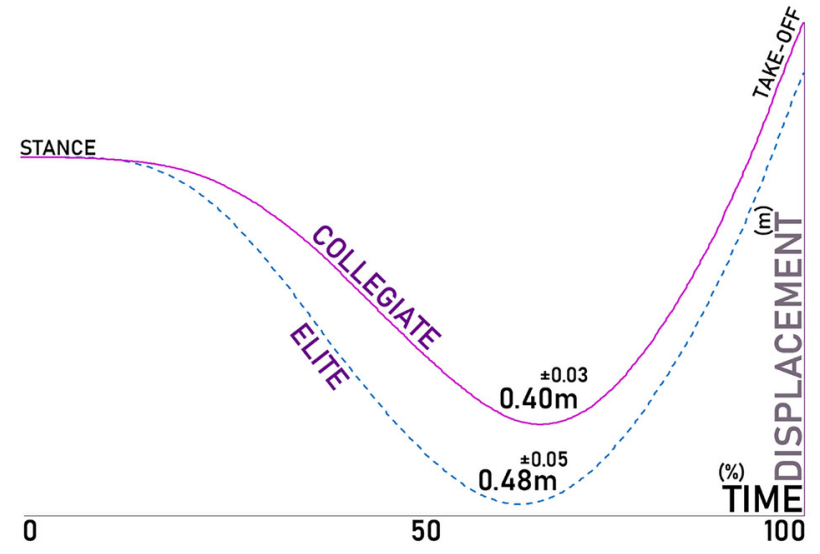

Figure 2. CoM Displacement before Take-Off

The correlations between F-T curve variables of eccentric and concentric phases and MRSI with $\mathrm{JH}$ among elite and collegiate players are shown in Table 2.

According to the Table 2, and despite entire eccentric variables, ETT exhibited a significant correlation with $\mathrm{JH}$ among total players $(\mathrm{r}=-0.58)$, albeit no strong correlation exists among groups. Of force variables, $\mathrm{CPF}$ was shown a significant correlation with collegiate $(\mathrm{r}=0.77)$ and total players $(\mathrm{r}=0.68)$. Significant correlation was also observed between CRPF and
$\mathrm{JH}$ in collegiate players $(\mathrm{r}=0.80)$, elites $(\mathrm{r}=0.69)$ and total players $(r=0.82)$. In contrast with elites, significant correlation was observed between CPP and JH in collegiate ( $r=0.56)$ and total players $(r=0.49)$. CRPP was the sole power variable that has a significant correlation among both elite $(r=0.83)$ and collegiate $(\mathrm{r}=0.79)$ groups and also total players $(\mathrm{r}=0.87)$. MRSI measures have also exhibited a strong correlation with $\mathrm{JH}$ in collegiate $(\mathrm{r}=0.71)$, elite $(\mathrm{r}=0.83)$ and total players $(\mathrm{r}=0.84)$.

Table 2. Correlation between force time variables of eccentric and concentric phases with ultimate jump height

\begin{tabular}{cccc}
\hline Variables & & JH & \\
& Collegiate (12) & Elite (12) & Total Participants (24) \\
\hline ETT & -0.47 & -0.23 & $-0.58^{*}$ \\
CTT & -0.05 & -0.12 & -0.10 \\
TtE & -0.14 & 0.08 & 0.03 \\
TtC & 0.08 & -0.05 & -0.02 \\
TtEPP & -0.12 & -0.04 & -0.16 \\
TtCPP & 0.18 & -0.15 & 0.07 \\
EPF & 0.42 & -0.18 & 0.29 \\
CPF & 045 & 0.49 & $0.56^{*}$ \\
ERPF & 0.12 & -0.26 & -0.15 \\
CRPF & $0.79^{* *}$ & $0.71^{*}$ & $0.80^{* *}$ \\
EPV & -0.22 & -0.11 & -0.29 \\
CPV & $0.98^{* *}$ & $0.99^{*}$ & $0.99^{* *}$ \\
EPP & 0.26 & 0.03 & 0.21 \\
CPP & $0.65^{*}$ & 0.38 & $0.53^{*}$ \\
EAP & 0.13 & -0.01 & 0.08 \\
CAP & $0.65^{*}$ & 0.23 & $0.49^{*}$ \\
ERPP & -0.09 & -0.01 & -0.12 \\
CRPP & $0.81^{* *}$ & $0.83^{* *}$ & $0.89^{* *}$ \\
MRSI & $0.83^{* *}$ & $0.71^{* *}$ & $0.81^{* *}$ \\
\hline
\end{tabular}

Legend: * Represents Moderate Correlation ${ }^{* *}$ Represents Strong Correlation

\section{Discussion}

This study was aimed to a) review the relationship between F-T curve variables of eccentric and concentric phases with $\mathrm{JH}$, and b) compare these variables among elite and collegiate basketball players. The main founding of this study was that elites performed the CMJ significantly faster than collegiate players and produced higher measures of force, velocity and consequently power. Collegiate players, on the other hand, produced higher amounts of relative force and power, which seems rational for the case that they owned a lower weight.
Correlation outcomes also reveals that ETT, CPF, CRPF. CPV, CPP, CRPP and MRSI had a significant correlation with ultimate $\mathrm{JH}$ among total basketball players.

\section{Time variables}

It has been pointed out that reducing the eccentric phase time could be used as a pragmatic manner leading to increases in the $\mathrm{JH}$ (Laffaye et al., 2014). Additionally, it is mentioned that a reduction in the eccentric phase time could lead to an increase in the muscle fibers recruitment, which in turn, could 
develop jump performance in order to obtain a higher jump (Sarvestan et al., 2018). Furthermore, based on force-velocity relationship, increases in the muscle lengthening contraction velocity result in the generation of additional force, and this can increase JH. As it is shown in Table 2, ETT exhibits significant negative correlation with $\mathrm{JH}$ among total participants, which is in line with previous studies mentioned above. This outcome indicates that a proper short eccentric phase provides muscle-tendon units with sufficient time for recruiting muscle fibers and help in employing the most possible fiber before depleting the calcium from sarcoplasmic reticulum. In terms of experience differences, elite players performed the CMJ, whether in eccentric or concentric phase, shorter than collegiate players. Based on this fact, it could be contemplated that elite players, based on their higher levels of experience, were aware of the proper timing in jump to reach the greater heights.

\section{Force variables}

Previous studies indicate that CPF is significantly correlated with JH (Laffaye et al., 2014; Pupo et al., 2012; Riggs \& Sheppard, 2009), which similar to the results of this study. As shown in Table 2, CPF is significantly correlated with $\mathrm{JH}$ among total participants. Mathematically, an increase in produced force leads to an increment in acceleration measures and, consequently, velocity measures during take-off. Hence, the higher take-off velocities, the greater jump heights. On the other hand, although CPF did not show any significant correlation with $\mathrm{JH}$ among elite and collegiate players, the number of participants must be taken into account as of research limitations in this study, and to this end, the total players statistical outcomes are more reliable for interpretation.

It is also reported that the CRPF is significantly correlated with JH (Pupo et al., 2012; Riggs \& Sheppard, 2009). Similarly, CRPF measures are significantly correlated with $\mathrm{JH}$ for elites $(\mathrm{r}=0.79)$, collegiate players $(\mathrm{r}=0.71)$, and total participants $(\mathrm{r}=0.80)$ in this study. Review on most studies conducted on CMJ revealed the scarcity of attention to the CRPF of athletes. Study on the relative measures of variables can provide a more precise criteria of athlete capability due to the nature of sports such as volleyball and basketball in which the ability of jumping and rapid movements are factors that affect performance, and the ability to overcome body weight or relative measures of force is potentially vital (Riggs \& Sheppard, 2009). Furthermore, although elites produced higher CPF in comparison with collegiate players, the amounts of CRPF were significantly higher in collegiate group, which seems rational because of lower weight of collegiate group.

\section{Velocity variables}

Elite players exhibited considerably higher concentric velocity measures compared with collegiate players $(\approx 0.23 \mathrm{~m} / \mathrm{s})$. Similarly, the eccentric velocity measures were greater in elite players, which seems to be the main reason for shorter ETT. It is observed that eccentric velocity is not significantly correlated with $\mathrm{JH}$ in both groups; however, concentric velocity is strongly correlated with $\mathrm{JH}$ among both groups and total players, which was predictable because of $\mathrm{JH}$ calculation formula ( $\mathrm{h}=\mathrm{v} 2 / 2 \mathrm{~g})$ (Sarvestan et al., 2018). Nevertheless, optimal utilization of SSC in elite players, resulted in higher COM velocities during eccentric and concentric phases and finally aided in greater measures of height.

\section{Power variables}

Irrespective of whether power is relative or absolute, it is considered as critical factor for athlete performance (Pupo et al., 2012; Sarvestan et al., 2018). Studies conducted on CMJ indicate that athlete peak power evidently affects JH (Pupo et al., 2012). Significant correlation between absolute peak power, relative peak power and average power measures with $\mathrm{JH}$ is reported formerly (Cronin \& Hansen, 2005; Pupo et al., 2012; Riggs \& Sheppard, 2009), which is similar to the outcomes of the present study. As it is depicted in Table 2, there is significant correlations between CPP, CAP and CRPP with JH for all participants. These results indicate that not only CPP, as a product of multiplication of force and speed, have an impact on ultimate $\mathrm{JH}$, but also individual power generated by each athlete could specifically influence $\mathrm{JH}$. Based on the outcomes, the importance of employing specific concentric exercises, such as plyometric or weightlifting, to improve force and power is obvious (Carlock et al., 2004; Markovic, 2007). In terms of comparison, elites produced higher amounts of CPP and CAP; However, collegiate players produced significantly greater amounts of CRPP, which is still because of their lower weight.

The results of the study highlight the importance of absolute and relative measures of force and power in order to achieve higher jumps among basketball players. Given that these variables and peak velocity of concentric phase undeniably affect the ultimate jump height, athletes could significantly rely on them and enhance their performance by focusing on the fore-mentioned factors in their training program. Elite players, in addition, performed CMJ in a shorter period of time with higher amounts of velocity. Kinematics and kinetics outputs are significantly affected by jump style. To this end, it should be noted that a few parameters, such as sport specific training, sport specific requirements, and players' experience levels, significantly impact Kinematics and kinetics parameters and lead to misinterpretations in designing a training program.

\section{Acknowledgements}

This study was financially supported by Internal Grant Agency (IGA) of Palacky university Olomouc (IGA-FTK-008). The authors of this study would like to thank the participants due to their commitment in the measurement procedure.

\section{Conflict of Interest}

The authors declare that there are no conflicts of interest.

Received: 15 February 2019| Accepted: 05 April 2019| Published: 01 June 2019

\section{References}

Bobbert, M.F., Gerritsen, K.G., Litjens, M.C., \& Van Soest, A.J. (1996). Why is countermovement jump height greater than squat jump height? Medicine and science in sports and exercise, 28, 1402-1412.

Bosco, C., Viitasalo, J., Komi, P., \& Luhtanen, P. (1982). Combined effect of elastic energy and myoelectrical potentiation during stretch-shortening cycle exercise. Acta Physiologica Scandinavica, 114(4), 557-565.

Canavan, P.K., \& Vescovi, J.D. (2004). Evaluation of power prediction equations: peak vertical jumping power in women. Medicine \& Science in Sports \& Exercise, 36(9), 1589-1593.

Carlock, J.M., Smith, S.L., Hartman, M.J., Morris, R.T., Ciroslan, D.A., Pierce, K.C., Newton, R.U., Harman, E.A., Sands, W.A., \& Stone, M.H. (2004). The relationship between vertical jump power estimates and weightlifting ability: a field-test approach. The Journal of Strength \& Conditioning Research, 18(3), 534-539.

Claudino, J.G., Cronin, J., Mezêncio, B., McMaster, D.T., McGuigan, M., Tricoli, V.Amadio, A.C., \& Serrão, J.C. (2017). The countermovement jump to monitor neuromuscular status: A meta-analysis. Journal of science and medicine in sport, 20(4), 397-402. 
Cormie, P., McBride, J.M., \& McCaulley, G.O. (2008). Power-time, force-time, and velocity-time curve analysis during the jump squat: impact of load. Journal of applied biomechanics, 24(2), 112-120.

Cormie, P., McBride, J.M., \& McCaulley, G.O. (2009). Power-time, force-time, and velocity-time curve analysis of the countermovement jump: impact of training. The Journal of Strength \& Conditioning Research, 23(1), 177186.

Cormie, P., McGuigan, M.R., \& Newton, R.U. (2010). Changes in the eccentric phase contribute to improved stretch-shorten cycle performance after training. Medicine \& Science in Sports \& Exercise, 42(9), 1731-1744.

Cronin, J.B., \& Hansen, K.T. (2005). Strength and power predictors of sports speed. J Strength Cond Res, 19(2), 349-357.

Jidovtseff, B., Quievre, J., Nigel, H., \& Cronin, J. (2014). Influence of jumping strategy on kinetic and kinematic variables. Journal of Sports Medicine and Physical Fitness, 54, 129-138.

Jiménez-Reyes, P., \& González-Badillo, J. (2011). Control de la carga de entrenamiento a través del CMJ en pruebas de velocidad y saltos para optimizar el rendimiento deportivo en atletismo.(Monitoring training load through the $\mathrm{CMJ}$ in sprints and jump events for optimizing performance in athletics). CCD. Cultura_Ciencia_Deporte. 文化-科技-体育 doi: 10.12800/ccd, 6 (18), 207-217.

Kollias, I., Hatzitaki, V., Papaiakovou, G., \& Giatsis, G. (2001). Using principa components analysis to identify individual differences in vertical jump performance. Research Quarterly for Exercise and Sport, 72(1), 63-67.

Laffaye, G., Wagner, P.P., \& Tombleson, T.I. (2014). Countermovement jump height: Gender and sport-specific differences in the force-time variables. The Journal of Strength \& Conditioning Research, 28(4), 1096-1105.

Markovic, G. (2007). Does plyometric training improve vertical jump height? A meta-analytical review. British journal of sports medicine, 41(6), 349-355.

McGinnis, R.S., Cain, S.M., Davidson, S.P., Vitali, R.V., Perkins, N.C., \& McLean, S.G. (2016). Quantifying the effects of load carriage and fatigue unde load on sacral kinematics during countermovement vertical jump with IMU-based method. Sports Engineering, 19(1), 21-34.

McMahon, J.J., Rej, S.J., \& Comfort, P. (2017). Sex differences in countermovement jump phase characteristics. Sports, 5(1), 8 .

Moir, G.L. (2008). Three different methods of calculating vertical jump height from force platform data in men and women. Measurement in Physical Education and Exercise Science, 12(4), 207-218.

Owen, N.J., Watkins, J., Kilduff, L.P., Bevan, H.R., \& Bennett, M.A. (2014). Development of a criterion method to determine peak mechanical power output in a countermovement jump. The Journal of Strength \& Conditioning Research, 28(6), 1552-1558.

Pupo, J.D., Detanico, D., \& Santos, S.G.D. (2012). Kinetic parameters as determinants of vertical jump performance. Revista Brasileira de Cineantropometric \& Desempenho Humano, 14(1), 41-51.

Riggs, M.P. \& Sheppard, J.M. (2009). The relative importance of strength and power qualities to vertical jump height of elite beach volleyball players during the counter-movement and squat jump.

Sarvestan, J., Cheraghi, M. Sebyani, M, Shirzad, E \& Svoboda, Z. (2018). Relationships between force-time curve variables and jump height during countermovement jumps in young elite volleyball players. Acta Gymnica, 48(1), 9-14.

Sheppard, J., McGuigan, M., \& Newton, R. (2008). The effects of depth-jumping on vertical jump performance of elite volleyball players: an examination of the transfer of increased stretch-load tolerance to spike jump performance. Journal of Australian Strength and Conditioning, 16(4), 3-10.

Slinde, F, Suber, C Suber, L, Edwén, C. E \& Svantesson, U. (2008). Test-retest reliability of three different countermovement jumping tests. The Journal of Strength \& Conditioning Research, 22(2), 640-644.

Smith, D., Roberts, D. \& Watson, B. (1992). Physical, physiological and performance differences between Canadian national team and universiade volleyball players. Journal of Sports Sciences, 10(2), 131-138. 